\title{
Glossopharyngeal Nerve and Vagus Nerve Palsies Associated with Influenza Vaccination
}

\author{
Kazuhiro Ishii, Tomomi Kanazawa, Yasushi Tomidokoro and Akira Tamaoka
}

\begin{abstract}
We herein report the first case of glossopharyngeal nerve and vagus nerve palsies that appeared after an influenza vaccination. A 15-year-old boy developed dysphagia and dysarthria seven days after receiving an inoculation of the inactivated influenza vaccine. Massive intravenous immunoglobulin (IVIg) treatment was applied, as the patient's symptoms were considered to be immunological adverse effects of the influenza vaccine. He responded well to IVIg, and the symptoms immediately diminished. The mechanisms underlying the development of neurologic symptoms following vaccination are difficult to determine; however, providing immediate immunological treatment, such as IVIg, is effective and beneficial in countering these symptoms.
\end{abstract}

Key words: glossopharyngeal nerve palsy, vagus nerve palsy, dysarthria, dysphagia, intravenous immunoglobulin (IVIg), postvaccination

(Intern Med 53: 259-261, 2014)

(DOI: 10.2169/internalmedicine.53.0687)

\section{Introduction}

Influenza vaccination is known to cause neurological disorders, such as Guillain-Barré syndrome (GBS), MillerFisher syndrome, transverse myelitis, acute disseminated encephalomyelitis (ADEM), seizures and cranial nerve palsy (1). Among postvaccination cranial nerve palsies, Bell's palsy (2) and abducens nerve palsy have been documented (3). Glossopharyngeal nerve and vagus nerve palsies; however, have not yet been reported. We herein report the first case of right glossopharyngeal nerve and vagus nerve palsies that appeared seven days after an influenza vaccination, causing dysphagia and dysarthria that improved immediately after intravenous immunoglobulin (IVIg) treatment.

\section{Case Report}

A healthy 15-year-old boy was inoculated with an inactivated influenza vaccine (KAKETSUKEN, Lot No.L71-B) via subcutaneous injection at his local clinic. The vaccine consists of inactivated A/California/7/2009 (H1N1) pdm09-, A/Victoria/210/2009 (H3N2)- and B/Brisbane/60/2008-like viruses. The patient had a past history of allergic reactions to steroids, including a urticarial rash and worsening of asthma, used to treat asthma.

No symptoms resembling the common cold, such as rash, fever, arthralgia or diarrhea, were observed immediately after the vaccination. However, the patient began to choke on his saliva and experienced difficulty in swallowing solid food seven days after vaccination. Twelve days after vaccination, his difficulty in swallowing was observed to have progressed and liquids began to be regurgitated into his nasal cavity while drinking. The patient's speech also became impaired. Twenty seven days after vaccination, he consulted a neurologist, who diagnosed right glossopharyngeal and vagus nerve palsies. He was subsequently admitted to our hospital on day 29 following vaccination.

On admission, the patient's breathing sounded normal, and no signs of fever, enlarged tonsils, lymph node swelling, hepatosplenomegaly or rashes were observed. A neurological examination found no abnormalities in the patient's visual acuity or visual field, and he maintained clear consciousness. Direct and indirect light reflexes were intact in both eyes, with normal eye movement without nystagmus or ptosis. The patient had unaffected hearing without tinnitus or rashes on the external acoustic meatus. No facial sensory 
impairments or facial paralysis were observed. The gag reflex was reduced with a positive curtain sign on the right side and right soft palate palsy. The patient exhibited a nasal voice, difficulty in swallowing and regurgitation of consumed liquids into the nasal cavity. The tendon reflexes were all normal without pathological reflexes. We observed no reductions in limb muscle strength, muscle tonus abnormalities, involuntary movements, sensory abnormalities, cerebellar ataxia or autonomic symptoms, such as tachycardia, bradycardia and hyper- or hypohidrosis.

A blood test showed that the patient's blood cell count, hepatic and renal functions and glucose tolerance were all normal. The blood serum $\operatorname{IgG}$, IgA and IgM and complement levels demonstrated no immunological abnormalities. A drug-induced lymphocyte stimulation test using the specific influenza vaccine 'lot' was strongly positive, at $346 \%$ (stimulation index <179\%). The patient's cerebrospinal fluid (CSF) was normal, with a cell count of $2 / \mu \mathrm{L}$, a protein level of $16 \mathrm{mg} / \mathrm{dL}$ and a glucose level of $61 \mathrm{mg} / \mathrm{dL}$. The IgG index was normal at 0.5 , no elevation of myelin basic protein was observed and oligoclonal bands were negative. The levels of angiotensin-converting enzyme and lysozyme in the serum and CSF were normal, and autoantibodies, such as antinuclear antibodies, proteinase-anti-neutrophil cytoplasmic antibody (PR3-ANCA), myeloperoxidase (MPO)-ANCA, SSA, SSB and anti-AchR antibodies, were negative. Both the IgG and IgM antibody titers against herpes simplex virus (HSV), varicellazoster virus (VZV), cytomegalovirus (CMV), EB virus (EBV), mycoplasma, influenza A (H1N1), (H3N2), B-1 and B-2 showed no significant alterations. All anti-ganglioside antibodies against GM1, GM2, GM3, GD1a, GD1b, GD3, GT1b, GQ1b, Gal-C, GalNAc-GD1a and GD1a/GD1b proteins were negative. Brain MR imaging, including diffusion-weighted imaging, T2-weighted imaging, fluid attenuated inversion recovery imaging and T1-weighted imaging with gadolinium enhancement, showed no abnormal signal changes in either the cranial nerves or brain. An extremity nerve conduction study (NCS) did not indicate conduction block, increased distal latency, reduced conduction velocity, vanishing $\mathrm{F}$ waves or $\mathrm{F}$ wave latency elongation. The results of a facial NCS were also normal bilaterally. A laryngeal fiberscope examination showed normal movement of the vocal cords without recurrent nerve paralysis. We diagnosed the patient with right glossopharyngeal nerve and vagus nerve palsies temporally associated with influenza vaccination.

The 32nd day after vaccination, we administered IVIg treatment $(0.4 \mathrm{~g} / \mathrm{kg} /$ day $)$ for five days because the patient had previously experienced an allergic reaction to steroid therapy. The treatment resulted in a prompt recovery from the dysphagia and dysarthria. On the 67th day after vaccination, we confirmed a complete recovery from the patient's difficulty in swallowing and speaking.

\section{Discussion}

According to a report by Williams S.E., neurological symptoms appear seven days after vaccination on average, Bell's palsy appears after nine days, optic neuritis appears after 14 days and abducens nerve palsy appears after 13 days. The findings observed in the current case are consistent with this earlier report. Referring to the WHO causality assessment criteria and modified Clinical Immunization Safety Assessment (CISA) criteria, vaccination is a possible cause of cranial nerve symptoms (1). It is difficult to prove a causal relationship between vaccination and neurological symptoms that appear after vaccination. However, due to the time sequence of the events (the symptoms manifested after vaccination), we believe that the vaccination itself was involved, at least temporally, in inducing the patient's neurological symptoms.

Only a few serious neurological adverse events caused by influenza vaccination have been reported in recent years; however, Bell's palsy (2), optic neuritis (4) and abducens nerve palsy (5) have been previously reported. In a cohort study, the occurrence of Bell's palsy increased significantly in association with early influenza vaccination (hazard ratio: 1.34) (6). Adverse neurological effects of vaccines other than the influenza vaccine include optic neuritis (7) and facial nerve palsy (8) caused by the anthrax vaccine, abducens nerve paralysis in children following measles, mumps, rubella (MMR) and diphtheria, pertussis, tetanus (DPT) vaccination (9) and oculomotor nerve paralysis in response to the MMR vaccine (10).

Cranial neuropathy develops as postvaccination GBS (11), and a number of GBS subtypes are characterized by isolated cranial nerve palsies without typical extremity paresis. Furthermore, cases of cranial neuropathy involving only the glossopharyngeal nerve and vagus nerve, in addition to bilateral facial palsies, have been reported as a pharyngo-facial variant (12) or multiple cranial neuropathy variant (13). Several cases of cranial neuropathy associated with glossopharyngeal nerve and vagus nerve palsies alone have also been reported (14). These studies reported the rapid improvement of symptoms in such cases following treatment with IVIg.

The pathogenesis of postvaccination cranial neuropathy is not clearly understood. Since the vaccine antigens and myelin proteins are similar in conformation, myelin proteins, such as myelin basic proteins, are thought to be responsible for the autoimmune response observed in such cases. In addition, considering the low incidence of these symptoms, it is possible that another antigenic factor interacts with the influenza antigen prior to the development of the immunological response (4).

The present study reported negative results for all known anti-ganglioside antibodies tested, thus supporting the latter hypothesis. It is also possible that an antibody that conformationally recognizes myelin proteins was synthesized or that the patient's autoimmune system responded to another 
myelin protein, thereby triggering focused demyelination of the glossopharyngeal and vagus nerves. The strong positive results obtained on the drug-induced lymphocyte stimulation test show that the patient was sensitized to this batch of vaccine. This phenomenon and the onset of symptoms after vaccination were adverse events resulting from an immune response to the influenza vaccine.

A major question, of course, is why only the glossopharyngeal nerve and vagus nerves were impaired. The motor branch of the glossopharyngeal nerve and vagus nerve that innervates muscles in the pharynx, larynx and upper part of the esophagus is derived from the nucleus ambiguous and is termed a special visceral efferent (SVE) due to its functional component. The SVE also includes the trigeminal nerve and facial nerve. It is interesting that the patient's impairments were largely confined to the trigeminal, facial, glossopharyngeal and vagus nerves in the aforementioned variant of GBS with multiple cranial neuropathy, suggesting the possible presence of specific myelin proteins for SVE. There are two possible reasons why the impairments were confined to the glossopharyngeal and vagus nerves in the present patient. The first reason is that the motor branches of both nerves are derived from the nucleus ambiguous and run in anatomical proximity to each other. The second reason is that both motor branches are functionally classified as SVE and the components of the myelin proteins of these nerves are likely to be similar to each other. It has been speculated that an immunological cross-reaction occurs between SVE-specific myelin proteins and the vaccine antigens (15).

Treatment for adverse neurological events that develop after vaccination includes steroids (2) and IVIg treatment (16), both of which are effective. We believe that the present case was caused by gross immunity against the cranial nerves due to vaccination, such as the development of GBS, and the glossopharyngeal nerve and vagus nerve palsies were cured following IVIg treatment. We consider that the present patient, who experienced an allergic reaction to steroids, had a subtype of postvaccination GBS accompanied by the impairment of the glossopharyngeal nerve and vagus nerves alone; therefore, we administered IVIg treatment in this case. The NCS results of the limbs in this case were normal, as were the findings of bilateral facial NCS. However, the patient's rapid recovery from dysphagia and dysarthria following IVIg treatment strongly suggests the presence of primarily demyelinating lesions. Cases of the cranial neuropathy variant type GBS with normal nerve conduction velocity (NCV) results in the limbs have also been reported (14).

To our knowledge, the present case involves the first report of glossopharyngeal nerve and vagus nerve palsies occurring in association with an influenza vaccination. It is therefore clear that influenza vaccination is a possible cause of glossopharyngeal nerve and vagus nerve palsies and that it is therefore important for clinicians to pay close attention to their patients. When such symptoms appear, immunological treatment, such as steroids and IVIg, should be administered immediately.

The authors state that they have no Conflict of Interest (COI).

\section{Acknowledgement}

The authors wish to thank Dr. Susumu Kusunoki from the Department of Neurology, Kinki University School of Medicine, Osaka, Japan for measuring the various serum anti-ganglioside antibodies.

\section{References}

1. Williams SE, Pahud BA, Vellozzi C, et al. Causality assessment of serious neurologic adverse events following 2009 H1N1 vaccination. Vaccine 29: 8302-8308, 2011.

2. Chou CH, Liou WP, Hu KI, Loh CH, Chou CC, Chen YH. Bell's palsy associated with influenza vaccination: two case reports. Vaccine 25: 2839-2841, 2007.

3. Leiderman YI, Lessell S, Cestari DM. Recurrent isolated sixth nerve palsy after consecutive annual influenza vaccinations in a child. J AAPOS 13: 317-318, 2009.

4. Hull TP, Bates JH. Optic neuritis after influenza vaccination. Am J Ophthalmol 124: 703-704, 1997.

5. Khan AO. Recurrent isolated sixth nerve palsy after consecutive annual influenza vaccination in a child. J AAPOS 13: 623, 2009.

6. Bardage C, Persson I, Ortqvist A, Bergman U, Ludvigsson JF, Granath F. Neurological and autoimmune disorders after vaccination against pandemic influenza A (H1N1) with a monovalent adjuvanted vaccine: population based cohort study in Stockholm, Sweden. BMJ 343: d5956, 2011.

7. Kerrison JB, Lounsbury D, Thirkill CE, Lane RG, Schatz MP, Engler RM. Optic neuritis after anthrax vaccination. Ophthalmology 109: 99-104, 2002.

8. Alp H, Tan H, Orbak Z. Bell's palsy as a possible complication of hepatitis B vaccination in a child. J Health Popul Nutr 27: 707708, 2009.

9. Yousuf SJ, Khan AO. Presenting features suggestive for later recurrence of idiopathic sixth nerve paresis in children. J AAPOS 11: 452-455, 2007.

10. Manzotti F, Menozzi C, Porta MR, Orsoni JG. Partial third nerve palsy after Measles Mumps Rubella vaccination. Ital J Pediatr 36: 59, 2010.

11. Yuki N. Guillain-Barré syndrome and anti-ganglioside antibodies: a clinician-scientist's journey. Proc Jpn Acad Ser B Phys Biol Sci 88: 299-326, 2012.

12. Unal-Cevik I, Onal MZ, Odabasi Z, Tan E. IVIG-responsive multiple cranial neuropathy: a pharyngo-facial variant of GuillainBarré syndrome. Acta Neurol Belg 109: 317-321, 2009.

13. Lyu RK, Chen ST. Acute multiple cranial neuropathy: a variant of Guillain-Barré syndrome? Muscle Nerve 30: 433-436, 2004.

14. Wang Q, Xiang Y, Yu K, Li C, Wang J, Xiao L. Multiple cranial neuropathy variant of Guillain-Barré syndrome: a case series. Muscle Nerve 44: 252-257, 2011.

15. Haines DE. An Overview of the Brainstem. In: FUNDAMENTAL NEUROSCIENCE for Basic and Clinical Applications. Fourth Edition. Elsevier, Philadelphia, PA, 2012: 140-143, and 186-188.

16. Choe YJ, Cho H, Bae GR, Lee JK. Guillain-Barré syndrome following receipt of influenza A (H1N1) 2009 monovalent vaccine in Korea with an emphasis on Brighton Collaboration case definition. Vaccine 29: 2066-2070, 2011.

(C) 2014 The Japanese Society of Internal Medicine http://www.naika.or.jp/imonline/index.html 